\title{
Drowsiness detection for single channel EEG by DWT best $\mathrm{m}$-term approximation
}

\author{
Tiago da Silveira, Alice de Jesus Kozakevicius, Cesar Ramos Rodrigues*
}

\begin{abstract}
Introduction: In this paper we propose a promising new technique for drowsiness detection. It consists of applying the best $\mathrm{m}$-term approximation on a single-channel electroencephalography (EEG) signal preprocessed through a discrete wavelet transform. Methods: In order to classify EEG epochs as awake or drowsy states, the most significant $m$ terms from the wavelet expansion of an EEG signal are selected according to the magnitude of their coefficients related to the alpha and beta rhythms. Results: By using a simple thresholding strategy it provides hit rates comparable to those using more complex techniques. It was tested on a set of 6 hours and 50 minutes EEG drowsiness signals from PhysioNet Sleep Database yielding an overall sensitivity (TPR) of $84.98 \%$ and $98.65 \%$ of precision (PPV). Conclusion: The method has proved itself efficient at separating data from different brain rhythms, thus alleviating the requirement for complex post-processing classification algorithms.
\end{abstract}

Keywords: Signal processing, Drowsiness detection, Wavelet transform, Best m-term approximation, Frequency bands, DB2 Wavelet.

\section{Introduction}

The World Health Organization (WHO) has been concerned with road traffic injuries for over four decades. According to WHO's report (Peden et al., 2004), around 1.2 million people die worldwide because of road traffic accidents every year. According to the same document, nearly $30 \%$ of fatal crashes involving heavy commercial transport in USA, and approximately $20 \%$ of commercial transport crashes in Europe were fatigue-related. In 2007 a similar scenario was revealed on the Brazilian federal highways, when 3.367 traffic accidents were also attributed to drowsiness (Bacchieri and Barros, 2011). Hence, drowsy driving is the major cause of hundreds of thousands of deadly accidents every year around the world. Being considered as a public health issue, a variety of countermeasures are currently under investigation to mitigate it.

Attempts to develop fatigue countermeasure EEG-based devices date back to 1993 (Hunn, 1993) when EEG was investigated as an assessment tool of fatigue in flight pilots. After that, researches addressing association between brain wave variations and driver drowsiness using EEG (Lal and Craig, 2001), and sleep stages classification methods as in (McKeown et al., 1998), have become increasingly frequent.

In a neurophysiological approach, drowsiness is known as the transition from awake into the sleeping

*e-mail: cesar@ieee.org

Received: 04 December 2014 / Accepted: 20 April 2015 state, characterized by decreased attention and slow movements. The sleep process starts with the activation and inhibition of neurons in different brain areas. As this process is rhythmic and synchronized, when observed through EEG signals it is identified by: (i) decreased activity of beta rhythm $([13-30] \mathrm{Hz})$; (ii) increase and subsequent reduction of alpha rhythm activity $([8-13] \mathrm{Hz})$; and (iii) increased theta rhythm activity ([4-8]Hz) (Blinowska and Durka, 2006).

In order to identify these variations in a realistic environment, Papadelis et al. $(2006 ; 2007)$ performed EEG measurements on drivers after normal sleeping or after remaining awake for at least 24 hours prior to the driving experiment. This study showed significant alterations in all frequency bands except gamma. The first minutes of the experiment revealed also the sensitivity in the beta and alpha bands with respect to the driver's drowsiness level. It is worth to notice that EEGs acquired from voluntary sleep in bed and involuntary sleep during driving exhibit differences in vertex and spindle waves morphologies (Yeo et al., 2007), nevertheless it is assumed that the main variations on beta, alpha and theta rhythms are present in every sleep context. Since those differences are restricted to specific EEG events while the proposed methodology considers only alpha and beta rhythms, this study developed from a voluntary sleep 
database is considered a reliable approach for a real world application.

Besides experimental knowledge, signal processing approaches have contributed to complement different aspects of the problem, treating drowsiness as a pattern to be recognized from brain signals. Previous studies commonly used Fast Fourier Transform (FFT) or Discrete Wavelet Transform (DWT) (Akay, 1998; Correa and Leber, 2010; De Carli et al., 1999; Liang et al., 2006; Subasi, 2005) as a feature selection technique, while Mahalanobis distance (Lin et al., 2010), independent component analysis (Lin et al., 2005) or neural network were used as classifiers (Subasi, 2005). Correa and Leber (2010) also used an artificial neural network to classify drowsiness through the extracted characteristics from an EEG signal using wavelets and Fourier spectrum. However, it is the multiresolution capability of the wavelet transform, providing a measure of EEG energy at each decomposition level, that makes it a better choice for automatic detection of arousals during the sleep (De Carli et al., 1999), as well as for many other applications using EEG and other biomedical signals (Akay, 1998).

Once dynamic power consumption of CMOS based processors is proportional to the product of supply voltage squared and clock frequency $\left(V_{d d}{ }^{2} \cdot f_{c k}\right)$ (Tan et al., 2014), the increasing processing power of portable devices is also incrementing the energy demand. In order to enable the implementation of EEG real-time processing on mobile, wearable or even implantable devices in a near future, the availability of both efficient and lightweight algorithms is a needful feature.

We therefore propose the application of best m-term approximation, a technique commonly considered for data compression, on wavelet transformed EEG signals as an alternative for identifying drowsiness patterns. The technique can be attractive for implementing fatigue detection on low-power devices because DWT kernels require fewer execution steps than FFT algorithms (complexities for transforming $1 D$ signals with $N$ points are respectively in the order of $N$ and $N . \log _{2}(N)$ steps (Beylkin et al., 1991)). Additionally, simple threshold techniques are employed instead of classification strategies based on heavy matrix operations (Lawhern et al., 2013; Liang et al., 2006; Lin et al., 2010). Another advantage of the proposed technique is the fact that no previous artifact rejection is necessary, since the thresholded wavelet representation of the EEG signal already works as a type of filtering process, removing low frequencies from the analyzed signal.
As a starting point for introducing the main tools of our analysis scheme, a short overview about the considered wavelet transform and the best m-term approximation strategy are summarized, followed by the proposed algorithm, the simulation results and the final discussion.

\section{Methods}

Wavelets have become an alternative and efficient tool to analyze EEG signals in association with other heuristics and methods. In (Sinha, 2008) wavelets were combined with neural networks for detecting sleep spindles, REM and wake states. In (Rosso et al., 2001) the relative wavelet energy provided information about the relative energy associated with different frequency bands present in the EEG. In the current work we consider the discrete wavelet transform firstly to recognize different brain rhythms and then to propose a heuristic to automatically detect the drowsiness state.

\section{Wavelet series expansion}

The wavelet analysis of a signal provides time and frequency information at different levels of resolution, representing also non-stationary signals (Daubechies, 1992). These characteristics justify the use of wavelets in the brain signal processing.

\section{Discrete Wavelet Transform}

The DWT can be understood as the discretization of the continuous wavelet transform (CWT). CWT consists of determining the coefficients of the wavelet series expansion of the signal $f(t)$, given by the correlation between $f(t)$ and the analyzing wavelet $\Psi(t)$.

$$
W f(\alpha, \tau)=f, \psi_{\alpha, \tau}=\int_{-\infty}^{+\infty} f(t) \overline{\psi_{\alpha, \tau}(t)} d t
$$

In Equation 1 the parameter $\alpha$ indicates the wavelet scale and the parameter $\tau$ indicates its position. The analysis is performed by varying these parameters continuously. On the other hand, DWT uses discrete values for $\alpha$ and $\tau$. The implementation of the DWT can be efficiently and accurately computed through filter banks (Mallat, 1989) when dyadic values are chosen for $\alpha$ and special restrictions are imposed to the wavelet family.

In this study, the filters considered are associated to the orthonormal with compact support functions from Daubechies wavelet family with two vanishing moments (DB2), implemented by the fast wavelet transform algorithm (Nielsen, 1998). The wavelet series expansion of the signal $f(t)$ for the DWT becomes 
$f(t)=\sum_{l=0}^{2^{J_{0}}-1} c_{J_{0}, l} \varnothing_{J_{0}, l}(t)+\sum_{j=J_{0}}^{J-1} \sum_{l=0}^{2^{j}-1} d_{j, l} \psi_{j, l}(t)$

For the Equation 2 and the others in this study, the following notation is used: $J_{0}$ is the coarsest level for the DWT, in which the inverse wavelet transform (IWT) starts, $J$ is the finest level with $2^{J}$ elements, equivalent to the input samples of the signal.

The coefficients set $\bar{f}=\left\{C_{J_{0}}, D_{J_{0}}, D_{J_{0}+1}, \ldots, D_{J-1}\right\}$ is obtained by applying the DWT. Each level $j$ has $2^{J}$ elements, $j=J_{0}, \ldots, J-1$, and therefore the total number of wavelet coefficients for the entire decomposition is $N=2^{J-1}-2^{j 0}$. The subset $C_{J 0}$ contains the scale coefficients $C_{J 0, l}$ for the level $J_{0}$ and positions $l=0,1, \ldots, 2^{\mathrm{J} 0}-1$. The subsets $D_{j}$ contain the wavelet coefficients $d_{j, l}$ for the level $j$ and positions $l=0,1, \ldots, 2^{j}-1$.

To allow multiresolution analysis of the signal $f$, the levels of DWT are ordered so that $J_{0} \leq j \leq J$. The choice of $J$ value depends on the signal length and the sample rate, while $J_{0}$ must be chosen with the highest possible value in order to minimize the border effects of the wavelet decomposition algorithm, when periodic boundary conditions are chosen.

\section{Relationship between scale and frequency}

Once drowsiness is characterized through brain rhythms, it is fundamental to establish a relationship between scales from the DWT decomposition and frequencies being considered intrinsic for the phenomena. Considering that each wavelet $\Psi(t)$ has a central frequency $f_{c}$ - which corresponds to the frequency of the sinusoid that best fits the waveform of $\Psi(t)$ - the relationship between scale and frequency can be given through the pseudo-frequencies $f_{\alpha}$, which relates $f_{c}$ to the scale $\alpha$ and to the sampling period $T_{s}$ (Misiti et al., 2010).

$f_{\alpha}=\frac{f_{c}}{\alpha T_{s}}$

Table 1 lists the pseudo-frequency equivalent to the main scales used in this study. Its values were computed with Equation 3 considering the sample period $T_{s}=0.01 \mathrm{~s}$, once the EEG signal was obtained with a sample frequency $f_{s}=100 \mathrm{~Hz}$.

Once the wavelets DB2 have compact support in time, their bandwidth cannot be limited (Strang and Nguyen, 1996), implying a spread frequency spectrum with some overlap between the DWT levels, as showed in Figure 1. However, this frequency response still allows to distinguish the main brain rhythms in an appropriate way for this study. The central frequency must be understood as a scale equivalence in the frequency domain, yet it might not be exact. To work with discrete wavelet transform in the frequency domain, the spectrum shown in Figure 1 must be analyzed jointly with Table 1. For example, the alpha rhythm comprises the frequencies between $8 \mathrm{~Hz}$ and $13 \mathrm{~Hz}$. Table 1 shows that, for a $J=10$ DWT, the central frequency of $8.33 \mathrm{~Hz}$ is equivalent to level $j_{7}$. Then looking at Figure 1 we see that the whole alpha rhythm spectrum best fits DB2 wavelet in the level $j_{7}$.

Further, the Daubechies Wavelet with two vanishing moments (DB2) was chosen, considering the fewer filters available, since it preserves the border values of the signal and introduces the smallest possible error on the borders of the analyzed signal, when dealing with the periodic form of the transform.

\section{Best m-term approximation}

Given $\bar{f}=\left\{C_{J_{0}}, D_{J_{0}}, D_{J_{0}+1}, \ldots, D_{J-1}\right\}$ the set of coefficients from the wavelet series expansion of $f(t)$, the best $\mathrm{m}$-term approximation chooses only the first $m$ wavelet coefficients of higher modulus from the total $N$ wavelet coefficients, with $m<N$ (Stollnitz et al., 1995; Temlyakov, 2003). The $N-m$ wavelet coefficients with lower magnitude are replaced by zero and the

Table 1. Relationship between scales, resolution level and frequency for DB2 wavelet $\left(f_{c}=0,6667, f_{s}=100 \mathrm{~Hz}\right)$.

\begin{tabular}{cccc}
\hline Scale & $\begin{array}{c}\text { Level } \\
(\boldsymbol{J}=\mathbf{1 2})\end{array}$ & $\begin{array}{c}\text { Level } \\
(\boldsymbol{J}=\mathbf{1 0})\end{array}$ & {$[\mathbf{H z}]$} \\
\hline 2 & $j_{11}$ & $j_{9}$ & 33.34 \\
4 & $j_{10}$ & $j_{8}$ & 16.67 \\
8 & $j_{9}$ & $j_{7}$ & 8.33 \\
16 & $j_{8}$ & $j_{6}$ & 4.17 \\
32 & $j_{7}$ & $j_{5}$ & 2.08 \\
64 & $j_{6}$ & $j_{4}$ & 1.04 \\
128 & $j_{5}$ & $j_{3}$ & 0.52 \\
\hline
\end{tabular}

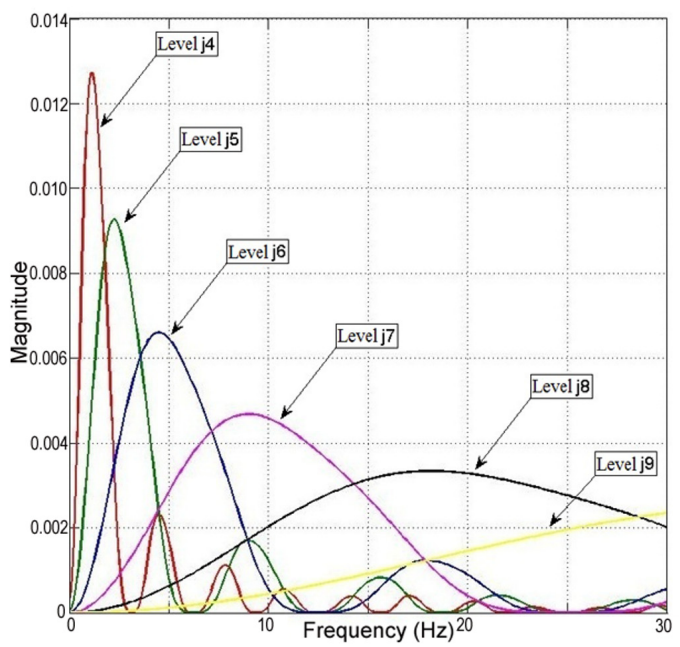

Figure 1. Wavelet DB2 frequency responses for a DWT $(\mathrm{J}=10)$ decomposition. 
scale coefficients $C_{j 0}$ are kept. The new set of modified coefficients is called $\bar{p}$ and its inverse wavelet transform (IWT) generates the approximated signal $p(t)$.

In fact, the number $m$ of active coefficients in the new expansion is chosen such that the approximation $p(t)$ of the signal $f(t)$ presents an error $p(t)-f(t)^{2}$ as small as possible, which is measured by the distance between the signal $f(t)$ and its approximation $p(t)$.

$\epsilon^{2}=p(t)-f(t)^{2}=\sqrt{\frac{\sum_{i=0}^{N-1}\left|p\left(t_{i}\right)-f\left(t_{i}\right)\right|^{2}}{N}}$

Due to orthonormality of the Daubechies wavelet basis, the error for the best m-term approximation method can be obtained as follows:

Let $\sigma(i)=(j, k), i=1,2, \ldots, m$, be the correspondence to the original level $j$ and position $k$ of the wavelet coefficients selected on the ordering process. In the same way, there is a correspondence $\rho(i)=(j, k)$ for all the non-significant coefficients, i.e. those replaced by zero. The square error for this approximation can be written just in function of the non-significant coefficients (Stollnitz et al., 1995).

$p(t)-f(t)^{2}=\sum_{i=1}^{N-m} d_{\rho(i)} \Psi_{\rho(i)}(t)^{2}=\sum_{i=1}^{N-m} d_{\rho(i)}$

Equation 5 shows that the minimum square error between the original function and the approximate function occurs when the wavelet coefficients are sorted in a decreasing way according to their magnitude, exactly as stated by the best m-term approximation method (Stollnitz et al., 1995).

What allows the application of this strategy to the drowsiness detection is that, according to numerical experiments from (Silveira, 2012), this operation maintains the frequency spectrum of the signal, even when $m \ll N$ (much smaller than). Furthermore, the wavelet transform time resolution is far superior when compared to the Short Time Fourier Transform (STFT), considering the levels associated to the higher frequencies (Mallat, 1989). To illustrate this, consider a signal $f(t)$ with the pure harmonic components of $5 \mathrm{~Hz}$ and $20 \mathrm{~Hz}$, as shown on Figure 2a. The significant coefficients after best m-term approximation $(\bar{p})$ are given on Figures $2 \mathrm{~b}$-d show the STFT analysis for $f(t)$ and $p(t)$, respectively.

A closer inspection of Figure $2 b$ shows that the best $\mathrm{m}$-term approximation further separates frequency contents. After applying the best m-term approximation, only significant coefficients from those DWT levels related to the frequency of input signal are preserved at each period of time.

\section{Drowsiness detection}

Through the joint analysis of Table 1 and Figure 1 we observe that there exists a connection between brain rhythms and DWT levels. Thus, considering an individual initially awake and alert, the drowsiness state is here characterized by (i) the absence of significant coefficients in levels that represent the

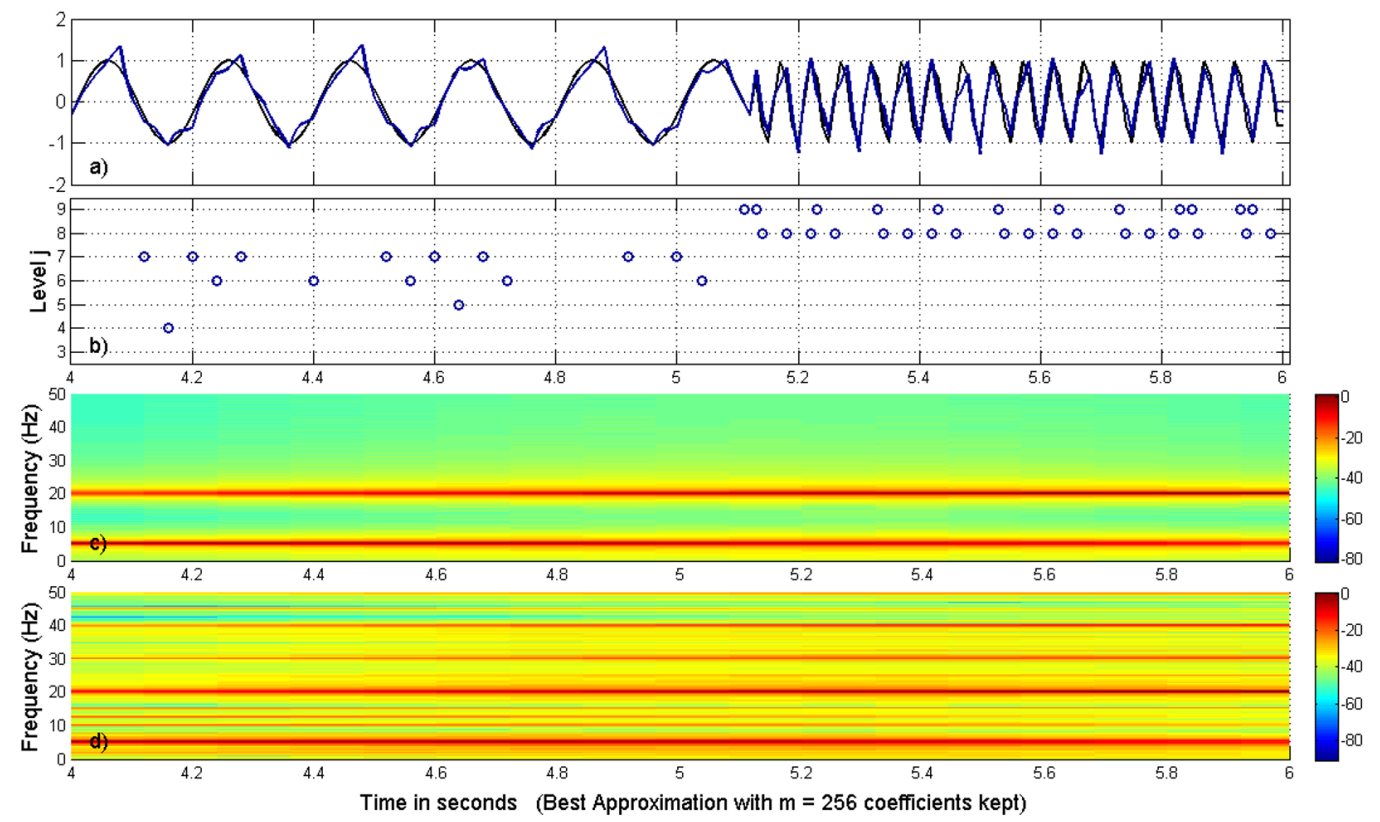

Figure 2. Best m-term approximation applied to a signal whose fundamental frequency is switched from 5 to $20 \mathrm{~Hz}$ at $5.1 \mathrm{~s}$. (a) $f(t)$ in black, $p(t)$ in blue. (b) Sparse representation of the significant coefficients $\bar{p}$. (c) STFT of $f(t)$. (d) STFT of $p(t)$. 
beta rhythm, $j_{\beta}$; (ii) concentration and a subsequent decrease of significant wavelet coefficients in levels related to alpha activity, $j_{\alpha}$, which means the person has closed his/her eyes in the moments preceding the drowsy state. The theta rhythm is represented by the lower DWT levels, which also represents noises and muscular movements. For this reason, the proposed algorithm considers, in the most analysis, just the alpha and beta rhythms.

Moreover, the best m-term approximation was applied to a study database (UniNova, 2010) in order to define the DWT levels that better represent each cognitive state. The signals were sampled at $f_{s}=100 \mathrm{~Hz}$ and analyzed in epochs of $30 \mathrm{~s}$ - each one zero-padded to 4096 samples, allowing a DWT with 12 levels. Considering these parameters, Table 2 shows the DWT level that corresponds to each brain rhythm.

Once it is known which brain rhythm is associated to the drowsiness process, this cognitive state can be detected by observing the DWT level that has more significant coefficients. Considering that each level $j$ of DWT has $2^{j}$ coefficients, the significant coefficients from levels $j_{\beta}$ and $\mathrm{J}_{\alpha}$ must be weighted.

$P_{\alpha}=\frac{m_{j_{\alpha}}}{2^{j_{\alpha}}} \cdot 100 ; P_{\beta}=\frac{m_{j_{\beta}}}{2^{j_{\beta}}} \cdot 100$

Where $m$ is the number of significant coefficients on each level indicated by the index $j_{\beta}$ or $j_{\alpha}$. $P_{\alpha}$, and $p_{\beta}$ are called alpha weight and beta weight, respectively.

Based on these statements, the following is proposed for analyzing EEG signals aiming to detect drowsiness characteristics:

Require: Signal $f(t)$ with $2^{J}$ samples; value of $m$ significant coefficients.

Ensure: AWAKE or DROWSY state.

$1: \operatorname{TWD}(\mathrm{f}) \rightarrow \bar{f}$

2: Best m-term approximation on $\bar{f} \rightarrow \bar{p}$

3: From $\bar{p}$, calculates $\mathrm{P}$

4: From $\bar{p}$, calculates $\mathrm{P}_{\beta}$

5: if $P_{\alpha}>\lambda_{\alpha}$ then

6: Calculates $\lambda_{\beta}\left(P_{\alpha}\right)$

7: if $P_{\beta}<\lambda_{\beta}$ then

8: State $\leftarrow$ DROWSY

9: else

10: State $\leftarrow$ AWAKE

11: end if

12: else

13: State $\leftarrow$ AWAKE

14: end if

The threshold parameters $\lambda_{\alpha}$ and $\lambda_{\beta}$ are obtained empirically when the proposed algorithm is applied to a training data. The training signals used in this study are from four patients of PhysioNet Sleep Database (Goldberger et al, 2000). The training
Table 2. Relationship among brain rhythms and DWT levels (DB2, $f=0,6667, f=100 \mathrm{~Hz}$ ).

\begin{tabular}{ccc}
\hline Brain rhythm & $\begin{array}{c}\text { DWT Level } \\
(\mathbf{J}=\mathbf{1 2})\end{array}$ & [Hz], DB2 \\
\hline Beta [13-30Hz] & $j_{\beta}=j_{11}$ & 33.34 \\
Alpha [8-13Hz] & $j_{\alpha}=j_{9}$ & 8.33 \\
Theta [4-8Hz] & $j_{\theta}=j_{8}$ & 4.17 \\
\hline
\end{tabular}

data contains 200 epochs of 30 s with the cognitive state evaluated on its hypnogram.

The steps 01 to 04 of the proposed algorithm were applied to each epoch of the training data in order to obtain the $P_{\alpha}$ and $P_{\beta}$ parameters. The results are shown in the feature space of Figure 3, where blue diamonds represent the epochs classified as the first sleep stage (N1) on the hypnogram, the purple circles represent the epochs considered as drowsy, while the red squares represent the epochs classified as awake on the hypnogram.

Analyzing the location of points in Figure 3, two classes of data can be distinguished: the red one, associated to awake state; and the blue one, associated to drowsiness. Based on this classification, the thresholding values are defined to limit the drowsiness region (bounded by the dashed green line). There are different ways to define mathematically this region. In this work, the simplest way is to, empirically, set a fixed $\lambda \alpha=25$. Performance can be optimized through the use of adaptive classifiers, at the cost of a higher computational burden, being beyond the scope of this paper. The value of $\lambda \beta$ can be obtained by the line equation calculated by interpolating points $\left(\mathrm{P}_{\alpha}=25\right.$; $\left.\mathrm{P}_{\beta}\right)$ and $\left(\mathrm{P}_{\alpha}=32 ; \mathrm{P}_{\beta}=5\right)$ the black line on Figure 3, whose equation is given by $\lambda_{\beta}=0.429 \mathrm{P}_{\alpha}-8.714$.

\section{Results}

To validate the algorithm proposed in the section before, 41 signals from 13 distinct patients of PhysioNet Sleep Database (Goldberger et al., 2000) were selected. Considering that each selected signal contains 20 epochs of 30 seconds, the algorithm was employed to evaluate a period equivalent to 6 hours and 50 minutes. The signals were collected from $F_{p z}$ channel (EEG electrode positioned at prefrontal region due to its ease of use in a driver facility, as in other studies (Hsu et al., 2013)) and sampled at $f_{s}=100 \mathrm{~Hz}$.

The signal selection was made in order to demonstrate the following cognitive states, based on the specialist hypnogram: (W) awake, with eyes opened and performing concentration activity; (D) awake but with closed eyes in a relaxed state, with 
EEG predominance of alpha rhythm (unlocked alpha) and (N1) corresponding to the first stage of sleep on R\&K classification (Avidan and Barkoukis, 2011).

Considering the cognitive states listed above, the selected signals were divided into three control groups:

A) The patient was awake during all the sampled period (W state).

B) The patient alternated between awake and relaxed state (W and D states).

C) The patient goes from an awake state to a relaxed state and then becomes sleepy (W, D and N1 states).

Drowsiness is then considered when the patient is on D or N1 states. However, the characteristics of each patient must be taken into account to interpret the results: the signals from Patient Sc4002e 0 and $\mathrm{Sc} 4112 \mathrm{e} 0$ contain all the cognitive states well defined and occurring in separate time. In turn, Patient $\mathrm{Sc} 4012 \mathrm{e} 0$ presents just a relaxed state before becoming drowsy, which leads to some false-positives, i.e., awake states identified as drowsy state. Patient Sc4102e0 has the absence of alpha activity (locked alpha), which is associated to cognitive and personal characteristics of the patient. In this case, drowsiness is detected mainly by the theta rhythm. All the other patients contains the group $\mathrm{C}$ characteristics.

For the sake of comparison, the metrics for awake and drowsy identifications was chosen based on confusion matrix in the same way as (Lin et al., 2010): True
Positive (TP - drowsy people correctly diagnosed as drowsy), False Positive (FP - alert people incorrectly identified as drowsy), True Negative (TN - alert people correctly identified as alert), and False Negative (FN - drowsy people incorrectly identified as alert). Hence, precision (PPV) and sensitivity (TPR) can be calculated as shown in Equation 7.

$P P V=\frac{T P}{T P+F P}, T P R=\frac{T P}{T P+F N}$

Where PPV represents the precision of drowsiness prediction, and TPR is the percentage of patients correctly identified as drowsy.

Based on this, Table 3 shows parameters of confusion matrix for each signal group, and for the whole set of epochs employed in this study.

\section{Discussion}

An increasing number of papers on EEG-based techniques for automatic fatigue detection can be found in literature. Proposed solutions comprehend combination of processing and classification techniques such as electronic filtering, FFT, WT and ANN, SVM, k-means and PCA. Nevertheless, devices for drowsiness detection and countermeasure, besides being the most pervasive as possible, must exhibit high reliability standards in order to prevent false alarms or undetected events. Hence, low-computational burden (and low-power consumption) allied to high hit rates are highly desirable features.

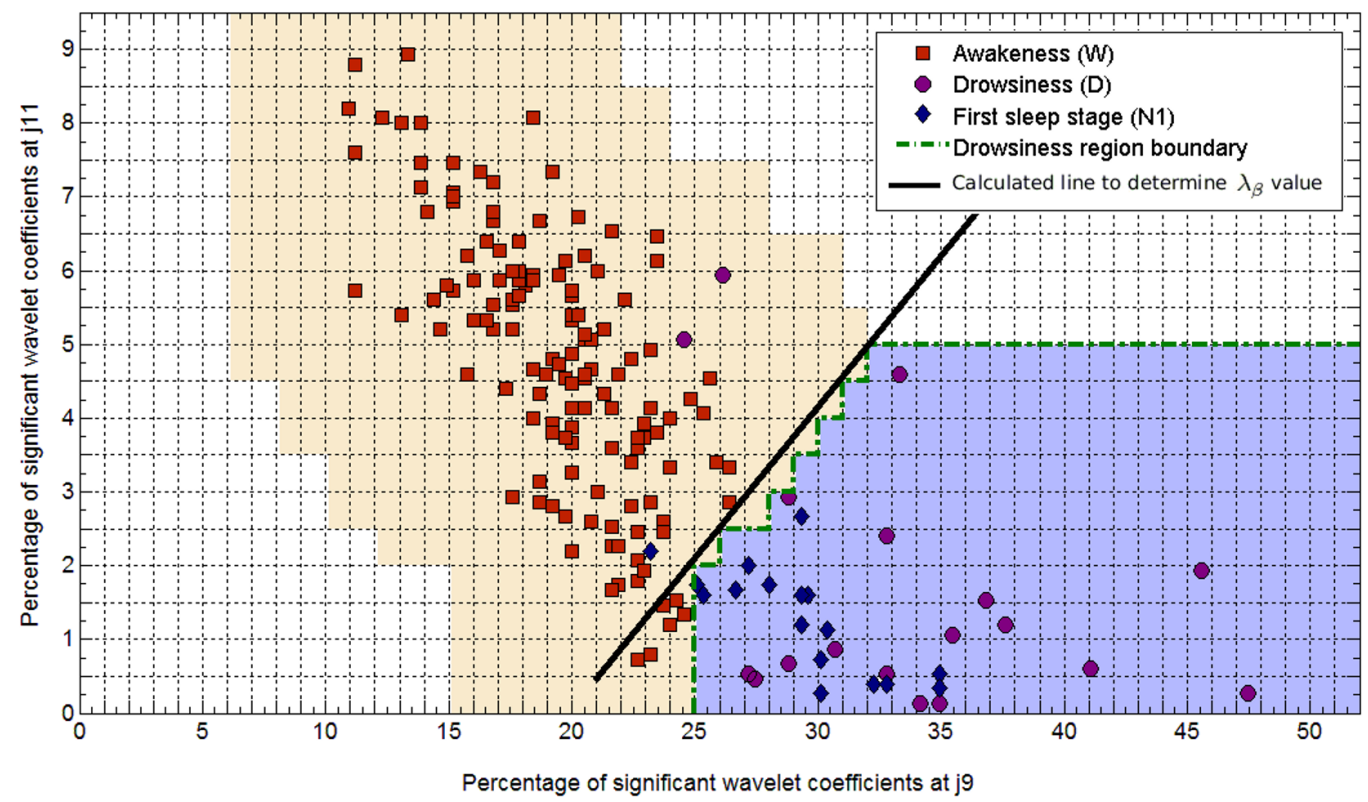

Figure 3. Drowsiness and awakeness classification via best m-term approximation. 
Table 3. Confusion matrix parameters considering the results when the algorithm is applied to the PhysioNet Sleep Database patients.

\begin{tabular}{|c|c|c|c|c|c|c|}
\hline Patient & TP (\%) & TN (\%) & FP $(\%)$ & FN (\%) & PPV (\%) & TPR (\%) \\
\hline $\mathrm{Sc} 4002 \mathrm{e} 0$ & 100 & 100 & 0 & 0 & 100 & 100 \\
\hline $\mathrm{Sc} 4012 \mathrm{e} 0$ & 100 & 78.69 & 21.31 & 0 & 82.43 & 100 \\
\hline $\mathrm{Sc} 4022 \mathrm{e} 0$ & 70 & 100 & 0 & 30 & 100 & 70 \\
\hline $\mathrm{Sc} 4032 \mathrm{e} 0$ & 40 & 100 & 0 & 60 & 100 & 40 \\
\hline $\mathrm{Sc} 4042 \mathrm{e} 0$ & 90 & 100 & 0 & 10 & 100 & 90 \\
\hline $\mathrm{Sc} 4052 \mathrm{e} 0$ & 100 & 100 & 0 & 0 & 100 & 100 \\
\hline $\mathrm{Sc} 4062 \mathrm{e} 0$ & 100 & 100 & 0 & 0 & 100 & 100 \\
\hline $\mathrm{Sc} 4072 \mathrm{e} 0$ & 80 & 100 & 0 & 20 & 100 & 80 \\
\hline $\mathrm{Sc} 4082 \mathrm{e} 0$ & 90 & 100 & 0 & 10 & 100 & 90 \\
\hline $\mathrm{Sc} 4092 \mathrm{e} 0$ & 70 & 100 & 0 & 30 & 100 & 70 \\
\hline $\mathrm{Sc} 4102 \mathrm{e} 0$ & 81.82 & 100 & 0 & 18.18 & 100 & 81.82 \\
\hline $\mathrm{Sc} 4112 \mathrm{e} 0$ & 82.86 & 100 & 0 & 17.14 & 100 & 82.86 \\
\hline $\mathrm{Sc} 4122 \mathrm{e} 0$ & 100 & 100 & 0 & 0 & 100 & 100 \\
\hline Total & 84.98 & 98.36 & 1.64 & 15.02 & 98.65 & 84.98 \\
\hline
\end{tabular}

Table 4. Comparison to other techniques.

\begin{tabular}{ccccc}
\hline Results & Sensitivity (\%) & Specificity (\%) & Accuracy (\%) & Precision (\%) \\
\hline (Lin et al., 2010) & 88.7 & - & - & 76.9 \\
(Hu et al., 2013) & 86 & 64 & 75 & - \\
(Lawhern et al., 2013) & 94.2 & 98.4 & - & 72.8 \\
This work & 99.9 & 97.5 & 98.7 & 97.4 \\
\hline
\end{tabular}

Our results are compared to results from other works in Table 4. Lin et al. (2010) have proposed a portable real-time microprocessed brain-computer interface (BCI) system for detecting drowsiness in drivers. They developed a detection algorithm that continuously computes the Mahalanobis distance between a set of alpha and beta rhythm amplitudes and an alertness reference model. In their work, alpha and beta components were obtained through a 512-point FFT. Hu et al. (2013) used a classification method based on support vector machine (SVM) on a collection of three-channel EEG signals for drive fatigue detection. EEG signals were preprocessed with an independent component analysis with reference (ICA-R) algorithm for electrooculography (EOG) interference removal. Another interesting set of results was presented by Lawhern et al. (2013): their detection strategy relied on the identification of alpha spindles employing discounted autoregressive (DAR) modeling.

Recently, some encouraging results were published reporting sensitivities higher than $88 \%$ (Lin et al., 2010) by allying FFT to Mahalanobis distance for classification of alpha and theta rhythms. Higher rates were reported by Lawhern et al. (2013). Hit rates higher than $94 \%$ were obtained by applying a sequential discounted autoregressive algorithm on a digital filtered EEG for detecting alpha spindles.
According to Blinowska and Durka (2006), the basic criterion for accepting an automatic method in the EEG analysis is its agreement to visual inspection of the hypnogram. According to the same authors, the correlation between the same classifications made by different specialists is $87.5 \%$ which is not high enough for implementing a safety system.

The analysis of results from Table 4 suggests that the proposed technique is promising for implementing drowsiness detection systems on portable devices, since discrete wavelet transform has lower computational cost when compared to FFT and the m-term best approximation seems to ease the identification job.

Furthermore, the proposed technique provides comparable sensitivities with a relative lower complexity. When applied to Daubechies WT, the best m-term approximation is able to select a set of coefficients highly correlated to alpha and beta rhythms. A simple thresholding strategy was sufficiently selective to reach precision rate higher than $98 \%$. Future works include testing it on a more comprehensive data set and investigating classification based on other parameters than alpha and beta rhythms.

\section{Acknowledgements}

The authors would like to thank to PPGI of Federal University of Santa Maria, to CNPq and FAPERGS, PG 1873-25.51/13-0. 


\section{References}

Akay M. Time Frequency and wavelets in biomedical signal processing. New York: IEEE Press; 1998.

Avidan AY, Barkoukis TJ. Review of sleep medicine: expert consult. 3rd ed. St. Louis: Saunders; 2011.

Bacchieri G, Barros AJD. Traffic accidents in Brazil from 1998 to 2010: many changes and few effects. Revista de Saude Publica. 2011; 45(5):949-63. http://dx.doi.org/10.1590/ S0034-89102011005000069. PMid:21953026.

Blinowska K, Durka P. Electroencephalography (EEG). New York: John Wiley; 2006.

Beylkin G, Coifman RRV, Rokhlin V. Fast wavelet transforms and numerical algorithms I. Communications on Pure and Applied Mathematics. 1991; 44(2):141-83. http://dx.doi. org/10.1002/cpa.3160440202.

Correa AG, Leber EL. An automatic detector of drowsiness based on spectral analysis and wavelet decomposition of EEG records. In: Annual International Conference of the IEEE; 2010 Aug 31-Sept 4; Buenos Aires, Argentina. Piscataway: IEEE; 2010. p. 1405-8. http://dx.doi.org/10.1109/ IEMBS.2010.5626721.

Daubechies I. Ten lectures on wavelets. Philadelphia: SIAM; 1992.

De Carli F, Nobili L, Gelcich P, Ferrillo F. A method for the automatic detection of arousals during sleep. Sleep. 1999; 22(5):561-72. PMid:10450591.

Goldberger AL, Amaral LAN, Glass L, Hausdorff JM, Ivanov PC, Mark RG, Mietus JE, Moody GB, Peng C-K, Stanley HE. PhysioBank, PhysioToolkit, and PhysioNet: components of a new research resource for complex physiologic signals. Circulation. 2000; 101(23):E215-20. http://dx.doi. org/10.1161/01.CIR.101.23.e215. PMid:10851218.

Hsu Y-L, Yang Y-T, Wang J-S, Hsu C-Y. Automatic sleep stage recurrent neural classifier using energy features of EEG signals. Neurocomputing. 2013; 104:105-14. http:// dx.doi.org/10.1016/j.neucom.2012.11.003.

$\mathrm{Hu}$ S, Peters B, Zheng G. Driver fatigue detection from electroencephalogram spectrum after electrooculography artifact removal. IET Intelligent Transport Systems. 2013; 7(1):105-13. http://dx.doi.org/10.1049/iet-its.2012.0045.

Hunn BP. The use of EEG as a workload assessment tool in flight test. California: Air Force Flight Test Center; 1993. Available from: http://www.dtic.mil/dtic/tr/fulltext/ u2/a274568.pdf.

Lal SKL, Craig A. Electroencephalography activity associated with driver fatigue: implications for a fatigue countermeasure device. Journal of Psychophysiology. 2001; 15(3):183-9. http://dx.doi.org/10.1027//0269-8803.15.3.183.

Lawhern V, Kerick S, Robbins KA. Detecting alpha spindle events in EEG time series using adaptive autoregressive models. BMC Neuroscience. 2013; 14(101):1. http://dx.doi. org/10.1186/1471-2202-14-101. PMid:24047117.

Liang S-F, Lin C-T, Wu R-C, Chen YC, Huang TY, Jung T-P. Monitoring driver's alertness based on the driving performance estimation and the EEG power spectrum analysis. In: Annual International Conference of the IEEE; 2006 Jan 17-18; Shangai, China. Piscataway: IEEE; 2006. p. 5738-41. http://dx.doi.org/ 10.1109/IEMBS.2005.1615791.

Lin C-T. Wu R-C, Liang S-F, Chao W-H, Chen Y-J, Jung T-P. EEG-based drowsiness estimation for safety driving using independent component analysis. IEEE Transactions on Circuits and Systems. 2005; 52(12):2726-38. http:// dx.doi.org/10.1109/TCSI.2005.857555.

Lin C-T, Chang C-J, Lin B-S, Hung S-H, Chao C-F, Wang I-J. A real-time wireless brain-computer interface system for drowsiness detection. IEEE Biomedical Circuits and Systems. 2010; 4(4):214-22. http://dx.doi.org/10.1109/ TBCAS.2010.2046415. PMid:23853367.

Mallat SG. A theory for multiresolution signal decomposition: the wavelet representation. IEEE Transactions on Pattern Analysis and Machine Intelligence. 1989; 11(7):674-93. http://dx.doi.org/10.1109/34.192463.

McKeown MJ, Humphries C, Achermann P, Borbély AA, Sejnowski TJ. A new method for detecting state changes in the EEG: exploratory application to sleep data. Journal of Sleep Research. 1998; 7(Suppl 1):48-56. http://dx.doi. org/10.1046/j.1365-2869.7.s1.8.x. PMid:9682194.

Misiti M, Oppenheim G, Poggi JM. Wavelet toolbox 4 user's guide. Natick: Mathworks; 2010.

Nielsen OM. Wavelets in scientific computing. Lyngby: Technical University of Denmark; 1998.

Papadelis C, Kourtidou-Papadeli C, Bamidis PD, Chouvarda I, Koufogiannis D, Bekiaris E, Maglaveras N. Indicators of sleepiness in an ambulatory EEG study of night driving. In: 28th Annual International Conference of the IEEE; 2006 Aug 30-Sep 03; New York, USA. Piscataway: IEEE; 2006. p. 5738-41. http://dx.doi.org/ 10.1109/IEMBS.2006.259614.

Papadelis C, Chen Z, Kourtidou-Papadeli C, Bamidis PD, Chouvarda I, Bekiaris E, Maglaveras N. Monitoring sleepiness with on-board electrophysiological recordings for preventing sleep-deprived traffic accidents. Clinical Neurophysiology : Official Journal of the International Federation of Clinical Neurophysiology. 2007; 118(9):1906-22. http://dx.doi. org/10.1016/j.clinph.2007.04.031. PMid:17652020.

Peden M, Scurfield L, Sleet D, Mohan D, Hyder AA, Jarawan E, Mathers C. World report on road traffic injury prevention. Geneva: World Health Organization; 2004. Available from: http://whqlibdoc.who.int/publications/2004/ 9241562609.pdf.

Rosso OA, Blanco S, Yordanova J, Kolev V, Figliola A, Schürmann M, Başar E. Wavelet entropy: a new tool for analysis of short duration brain electrical signals. Journal of Neuroscience Methods. 2001; 105(1):65-75. http://dx.doi. org/10.1016/S0165-0270(00)00356-3. PMid:11166367.

Silveira, T. Drowsiness detection from a single electroencephalography channel through discrete wavelet transform [dissertation]. Santa Maria: Federal University of Santa Maria; 2012.

Sinha RK. Artificial neural network and wavelet based automated detection of sleep spindles, REM sleep and wake states. Journal of Medical Systems. 2008; 32(4):291-9. http:// dx.doi.org/10.1007/s10916-008-9134-z. PMid:18619093. 
Stollnitz EJ, Derose TD, Salesin DH. Wavelets for computer graphics: a primer, part 1. IEEE Computer Graphics and Applications. 1995; 15(3):76-84. http://dx.doi. org/10.1109/38.376616.

Strang G, Nguyen T. Wavelets and filter banks. Wellesley: Wellesley College; 1996.

Subasi A. Automatic recognition of alertness level from EEG by using neural network and wavelet coefficients. Expert Systems with Applications. 2005; 28(4):701-11. http://dx.doi.org/10.1016/j.eswa.2004.12.027.

Tan L, Kothapalli S, Chen L, Hussaini O, Bissiri R, Chen Z. A survey of power and energy efficient techniques for high performance numerical linear algebra operations.
Parallel Computing. 2014; 40(10):559-73. http://dx.doi. org/10.1016/j.parco.2014.09.001.

Temlyakov VN. Nonlinear methods of approximation. Foundations of Computational Mathematics. 2003; 3(1):33107. http://dx.doi.org/10.1007/s102080010029.

UniNova. Sleep electroencephalography signal database. Lisbon: UNINOVA; 2010.

Yeo MV, Li X, Wilder-Smith EP. Characteristic EEG differences between voluntary recumbent sleep onset in bed and involuntary sleep onset in a driving simulator. Clinical Neurophysiology : Official Journal of the International Federation of Clinical Neurophysiology. 2007; 118(6):1315-23. http:// dx.doi.org/10.1016/j.clinph.2007.02.001. PMid:17398150.

\section{Authors}

Tiago da Silveira ${ }^{1}$, Alice de Jesus Kozakevicius ${ }^{2}$, Cesar Ramos Rodrigues ${ }^{3 *}$

${ }^{1}$ Programa de Pós-Graduação em Informática - PPGI, Universidade Federal de Santa Maria - UFSM, Santa Maria, RS, Brazil.

${ }^{2}$ Laboratório de Análise Numérica e Astrofísica - LANA, Departamento de Matemática, Universidade Federal de Santa Maria - UFSM, Santa Maria, RS, Brazil.

${ }^{3}$ Departamento de Eletrônica e Computação - DELC, Universidade Federal de Santa Maria - UFSM, CEP 97105-900, Santa Maria, RS, Brazil. 